\title{
Estado poblacional del orden Plecoptera (Insecta) en el Parque Nacional Sierra Nevada en Venezuela y sus implicaciones para planes de conservación
}

\author{
Maribet Gamboa \\ Leibniz-Institut of Freshwater Ecology and Inland Fisheries (IGB), Deparment of Shallow Lakes and Lowland Rivers, \\ Müggelseedamm 301, 12587 Berlin, Germany; gamboa@igb-berlin.de, maribetg@gmail.com
}

Recibido 26-III-2010. Corregido 20-VI-2010. Aceptado 26-VII-2010.

\begin{abstract}
Population status of insects of Plecoptera order in Sierra Nevada National Park in Venezuela and its implications for conservation planning. Longitudinal distribution of Plecoptera species were examined along the Sierra Nevada National Park in the Andean region of Merida State, Venezuela. PNSN is one of the largest protected areas and consists of two major sub-basins. Quantitative samples were collected in 7 river tributaries along the PNSN from February to May of 2009, and a total of 135 individuals and 4 species of the genus Anacroneuria were collected. Only three rivers (Nuestra Señora, La Picón y Corcovada) found the presence of stoneflies, the principal component analysis show that the characterization of habitat, current velocity, dissolved oxygen and the absence of human disturbance sources of influence in a suitable habitat for populations. Stoneflies are endangered or have become extinct throughout much of its range due to human activities. Conservation plans must be implemented urgently, in order to avoid recreational areas and/or economic in vicinity thereof. Rev. Biol. Trop. 58 (4): 1299-1310. Epub 2010 December 01.
\end{abstract}

Key words: Andean rivers, Anacroneuria, longitudinal gradient, environmental relationships, human activities.

El manejo de los sistemas acuáticos deriva de cambios producidos en el ambiente (Hurtado et al. 2005). Estos cambios remarcan un paralelismo existente entre la sucesión ecológica, las características físicas-químicas del cauce y la organización espacial de los ríos (Margalef 1983), al igual que en la distribución de poblaciones animales.

Diversos autores han estudiado la distribución espacial de los insectos bentónicos (Lillehammer \& Brittain 1987, Domínguez \& Valdez 1992, López \& Membiela 1993, Death \& Winterbourn 1995, Albariño 1997, Froehlich \& Oliveira 2002, Miserendino 2006 , entre otros) y sugieren que estos insectos proveen una importante información de las condiciones ambientales, y la respuesta de los organismos a las condiciones del hábitat lótico (Margalef 1983).
Los insectos que habitan en ambientes lóticos, incluyendo a los plecópteros están sujetos a las perturbaciones naturales y las producidas por el hombre (Miserendino 2006). Los factores naturales, como la temperatura, elevación, latitud, tipo de sustrato, descarga y velocidad de la corriente, son relativos a la geología, clima y vegetación de una región particular y son los factores primarios, los que determinan la distribución de los plecópteros (Hynes 1970, Cummins \& Klug 1979, Reice 1980, Ward \& Stanford 1982, Statzner et al. 1988, Dudgeon 1993, Townsend et al. 1997).

Las perturbaciones producidas por el hombre sobre los sistemas acuáticos, se deben principalmente a la introducción de especies exóticas, la construcción de reservas o diques, la deforestación que ocasiona aumento de sedimentación, la descarga de sustancias tóxicas 
(efluentes industriales, fertilizantes) y orgánicas (agricultura, urbanización) (Rabeni \& Minshall 1977, Resh et al. 1988, Death \& Winterbourn 1995). Diversos estudios explican cómo estos impactos afectan a las comunidades acuáticas, por lo tanto Plecoptera es uno de los grupos más sensibles a la perturbación (Merritt \& Cummins 1996, Miserendino 2000).

En los sistemas lóticos, se ha demostrado que los plecópteros, pueden ser una fuerza importante para estructurar las comunidades animales, ya que regulan de distintas maneras la distribución y abundancia de los organismos acuáticos. Directamente, causan una reducción en la abundancia numérica y en biomasa de las poblaciones de presas, lo cual, tendría consecuencias significativas a nivel de la comunidad, pudiendo llevar a la eliminación local de una especie. Indirectamente, podrían afectar la estructura de la comunidades acuáticas, al causar: i) alteraciones significantes en los patrones espacio-temporales de la actividad y distribución de las presas; ii) reacciones en cadena, como el efecto de "cascada top-down", cuando el consumo de una determinada presa puede afectar a componentes inferiores de la cadena trófica, así como a sus competidores; iii) restricciones en el uso del hábitat y en el comportamiento diario de forrajeo; y iv) cambios en los patrones del ciclo de vida (crecimiento y fecundidad) inducidos por la presencia de un depredador (Peckarsky 1993, Allan 1995, Giller \& Malmqvist 1998).

Los plecópteros han sido usados en el biomonitoreo de la contaminación orgánica (Merritt \& Cummins 1996) y también como parte del grupo EPT (Ephemeroptera-Plecoptera-Trichoptera) en la evaluación del efecto de la contaminación en los ríos, la deforestación y conversión de pastizales (Scarsbrook \& Halliday 1999, Harding et al. 2000). Algunos plecópteros son afectados por la regulación del río (Brittain 1991) y su riqueza decrece cuando disminuye la diversidad del hábitat (Landa et al. 1997).

En 1992, la IUCN (International Union for Conservation of Nature) categorizó a los plecópteros: Alloperla roberti como especie extinta, Leptoperla cacuminis y Riekoperla darlingtoni como especies vulnerables y Eusthenia nothofagi como especie con data insuficiente en Estados Unidos y Australia, respectivamente (IUCN 2009). Por lo tanto es necesario conocer el estado poblacional actual de los plecópteros en regiones templadas y tropicales.

En la región Neotropical, el orden Plecoptera está representado en su mayoría por la familia Perlidae, dentro de la cual Anacroneuria es el género predominante y se encuentra ampliamente distribuido en Venezuela, con 34 especies conocidas para el país, de las cuales 9 presentan distribución en la Cordillera Andina (Cressa \& Stark 2003).

Existe muy poca información acerca de los plecópteros en Venezuela y especialmente de su distribución dentro de la Cordillera Andina Venezolana. Hasta el momento el aporte más importante que se ha hecho para su conocimiento en esta área, proviene del estudio realizado por Maldonado (2002), quien evaluó la distribución de los plecópteros a nivel de especies y su relación con un conjunto de variables ambientales, en ríos de la Cordillera Andina, donde señala que menos del 5\% de las especies están estudiadas con respecto a su biología y distribución.

El Parque Nacional Sierra Nevada, es uno de los parques naturales más grandes del estado Mérida en Venezuela, tiene gran importancia desde el punto de vista de la producción hídrica, ya que muchas de sus cuencas hidrográficas constituyen las fuentes de abastecimiento de agua para el consumo humano. El parque esta sujeto a múltiples actividades y usos, incluyendo la agricultura, irrigación y generación de electricidad, por lo que provee una oportunidad para el estudio de los patrones de distribución de plecópteros y sus relaciones con el ambiente.

El objetivo de este estudio es: (1) Analizar la distribución de las especies de plecópteros e (2) identificar las posibles variables que influyen en los patrones de la comunidad a lo largo del parque en los principales ríos tributarios. 


\section{MATERIALES Y MÉTODOS}

Sitio de estudio: El Parque Nacional Sierra Nevada (PNSN) abarca una superficie 276.446 ha y está situado en pleno núcleo de la Cordillera de Mérida ( $8^{\circ} 10^{\prime} 38^{\prime \prime}-8^{\circ} 53^{\prime} 08^{\prime \prime}$ $\left.\mathrm{N}, 70^{\circ} 32^{\prime} 50^{\prime \prime}-71^{\circ} 17^{\prime} 04^{\prime \prime} \mathrm{W}\right)$, perteneciente a la Región Andina de Venezuela. Se localiza casi íntegramente en el macizo Sierra Nevada de Mérida, y está separado del macizo Sierra de La Culata por la depresión longitudinal del río Chama que le sirve de límite norte; mientras que por el noreste lo hace el fondo del valle del río Santo Domingo. Por el suroeste, el límite del parque lo conforma el fondo del valle del río Nuestra Señora y por el sur tiene como límites los bajos contrafuertes del macizo de la Sierra Nevada y la Sierra de Santo Domingo (Pérez 2000).

El territorio perteneciente al PNSN se caracteriza por presentar dos patrones de precipitación según la exposición de sus vertientes y el rango de temperatura oscila su media anual entre los $26^{\circ} \mathrm{C}$ en las áreas mas bajas (400m.s.n.m.) y $\operatorname{los}-0^{\circ} \mathrm{C}$ en las máximas alturas (>4 900 m.s.n.m.) (Fundación Andígena 2009).

La vegetación principal está representada por bosques ombrófilos montanos siempre verdes, que ocupan la faja altitudinal comprendida entre los 2000 y 3 000m.s.n.m, las especies arbóreas dominantes son: el quindú (Laplacea fructicosa), el say-say (Weinmannia jahni), el laurel (Ocotea sp.), entre otras. A partir de los $3000 \mathrm{~m}$ de altitud se desarrolla la vegetación de páramo, constituida por comunidades herbáceas y arbustivas dominada por especies de frailejón (Espeletia). También se presenta una vegetación arbustal xerofítica en las áreas más secas de la Cuenca Río Nuestra Señora. (Pérez 2000)

El parque está integrado por 13 cuencas y subcuencas hidrográficas principales a lo largo de sus dos vertientes; de estas cuencas, dos drenan sus aguas hacia la Hoya del Lago de Maracaibo a través de los ríos Chama y Nuestra Señora; las restantes once cuencas drenan sus aguas hacia la gran Hoya del Río Apure (Fundación Andígena 2009).

Siete ríos tributarios tipo II que drenan en el PNSN fueron muestreados (Fig. 1), La Mucuy ( $\left.8^{\circ} 37^{\prime} 13^{\prime \prime} \mathrm{N}, 71^{\circ} 04^{\prime} 02^{\prime \prime} \mathrm{W}\right)$; Nuestra Señora $\left(8^{\circ} 27^{\prime} 32.1^{\prime \prime} \mathrm{N}, 71^{\circ} 4^{\prime} 11.9^{\prime \prime} \mathrm{W}\right)$; La Picón ( $\left.8^{\circ} 37^{\prime} 56,2^{\prime \prime} \mathrm{N}, 71^{\circ} 02^{\prime} 12,3^{\prime \prime} \mathrm{W}\right)$; Aracay ( $\left.8^{\circ} 54^{\prime} 54.3^{\prime \prime} \mathrm{N}, 70^{\circ} 37^{\prime} 16,4^{\prime \prime} \mathrm{W}\right)$; Santo Domingo ( $8^{\circ} 46^{\prime} 56.0^{\prime \prime} \mathrm{N}, 76^{\circ} 48^{\prime} 17.7^{\prime}$ ' W); Las Piñuelas $\left(8^{\circ} 40^{\prime} 32.1^{\prime \prime} \mathrm{N}, 70^{\circ} 55^{\prime} 21.7^{\prime \prime} \mathrm{W}\right)$; y Corcovada ( $\left.8^{\circ} 47^{\prime} 17.4^{\prime \prime} \mathrm{N}, 70^{\circ} 48^{\prime} 53.9^{\prime \prime} \mathrm{W}\right)$, los cuales representan las principales afluentes dentro de las principales microcuencas (La Fría y Nuestra Señora).

Metodología de campo y laboratorio: En cada uno de los siete ríos muestreados, se recolectaron ninfas de plecópteros mediante una red de patada (o D-net), de $0.5 \mathrm{~mm}$ de tamaño del poro y $1 \mathrm{~m}^{2}$ de extensión, este tipo de muestreo consiste en colocar la red contracorriente y remover el sustrato del fondo bajo la extensión de la red, $1 \mathrm{~m}^{2}$, a fin de que los organismos sean arrastrados por la corriente al interior de la red. Se realizaron 12 esfuerzos de muestreo de 10min cada uno, mensualmente durante Febrero, Marzo, Abril y Mayo del 2009, con un total de 48 muestras para cada río y 336 muestras para todo el período.

Las muestras fueron guardadas a $95 \%$ de etanol e identificadas mediante las descripciones de Stark (1999, 2000), Maldonado (2002) y Maldonado et al. (2002).

En cada río se midieron in situ variables físicas, químicas y morfométricas según Maldonado (2002) y Segnini (2003):

- Caracterización química del agua: temperatura del agua, temperatura del ambiente y $\mathrm{pH}$, con un pHmetro de campo Sharp waterproof pH52 Meter. La salinidad (ppm), conductividad $(\mu \mathrm{S} / \mathrm{cm})$ y sólidos totales disueltos - TDS (ppm), con Tracer 1749 LaMotte. La dureza (gpg CaCO3) con Hardness Kit 4482-DR-LI LaMotte. El oxígeno disuelto (ppm) con Dissolved Oxigen kit 7414 LaMotte. Y el nitrito 


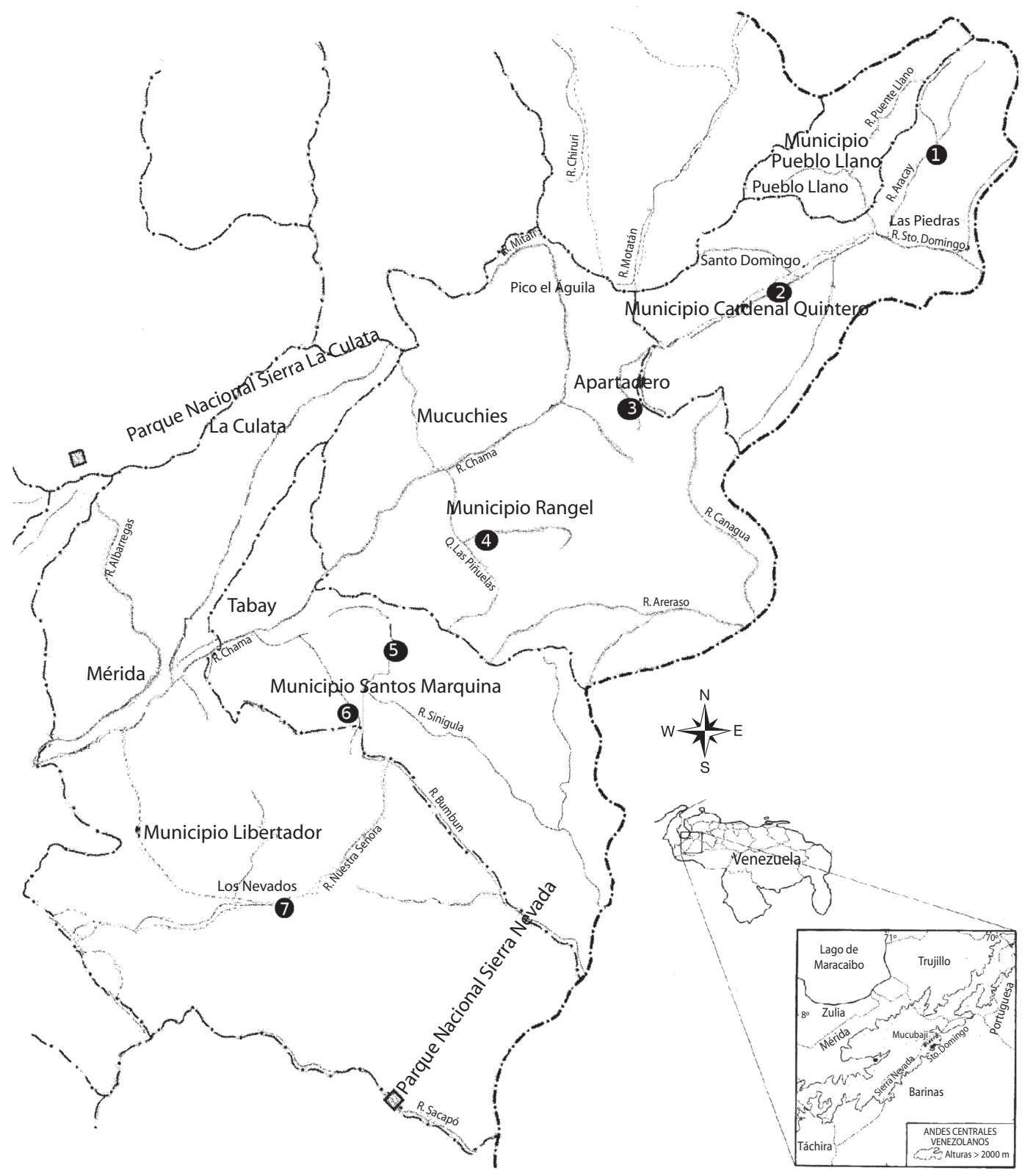

Fig. 1. Localidades de muestreo, Parque Nacional Sierra Nevada, Mérida, Venezuela. Donde: 1. Río Aracay; 2. Río Santo Domingo; 3. Quebrada Corcovada; 4. Quebrada Las Piñuelas; 5. Río La Mucuy; 6. Río La Picón; 7. Río Nuestra Señora.

Fig. 1. Study area of the 7 sampling localities in the Sierra Nevada National Park, Mérida, Venezuela. Where: 1. Aracay river, 2. Santo Domingo river, 3. Corcovada river, 4. Las Piñuelas river, 5. La Mucuy river, 6. La Picon river, 7. Nuestra Señora river. 
(ppm) y el nitrato (ppm), con Insta kit $2996\left(\mathrm{NO}_{2}\right.$ y $\left.\mathrm{NO}_{3}\right)$ LaMotte.

- Caracterización física del agua: ancho de la corriente $(\mathrm{m})$, profundidad media $(\mathrm{cm})$, velocidad de la corriente $(\mathrm{m} / \mathrm{s})$, mediante un flotador se determinó el tiempo que tarda un determinado recorrido y altura máxima del cauce (m), con un metro de campo. La elevación y coordenadas del punto de muestreo con GPS Garmin Geko 301.

- Caracterización morfométrica: consistió en la identificación de la zona mediante claves sobre las características de la corriente y del cauce, componentes inorgánicos del suelo, vegetación ribereña y características del hábitat (Segnini 2003).

Se realizó el análisis de componentes principales (ACP) con el programa estadístico Past versión 1.42 (Hammer et al. 2001), mediante $\log \mathrm{x}+1$ para demostrar qué variable ambiental tiene relación con la presencia y/o distribución de plecópteros en el río. Se midió el largo total y el ancho cefálico según Gamboa y Arrivillaga (en prensa) en todos los individuos de cada especie recolectados. Posteriormente se colocaron estos organismos en una estufa a $60^{\circ} \mathrm{C}$ por $24 \mathrm{~h}$, para luego ser pesados en una balanza analítica (precisión $0.001 \mathrm{mg}$ ) y así determinar el peso seco de los individuos. Las medidas corporales y el peso seco entre especies se compararon empleando una prueba T-student con un nivel de significancia de 0.05 .

\section{RESULTADOS}

Caracterización ambiental: La temperatura del ambiente y el tamaño del sustrato fueron similares para todos los ríos (Cuadro 1), donde oscila entre 20 a $30^{\circ} \mathrm{C}$ la temperatura dependiendo de la época y hora del día muestreada, y los sustratos tipo piedra o roca fueron los que dominaron las características del cauce. La temperatura del agua osciló entre los 9.5 a $17.6^{\circ} \mathrm{C}$ y se convirtió en un parámetro clave para el estudio de poblaciones de insectos acuáticos.

Las variables químicas medidas in situ (Cuadro 2), proveen una distinción ecológica entre los ríos estudiados. El río La Mucuy presenta mayores valores de dureza, salinidad, y sólidos disueltos en suspensión (TDS), como consecuencia de la presencia de zonas urbanas y recreativas al igual que el río Las Piñuelas. El río Aracay y Santo Domingo cuyo contenido de nitritos y nitratos, indica la presencia de ganadería en las cercanías del mismo. Y los ríos La Picón, Nuestra Señora y Corcovada,

CUADRO 1

Medidas ambientales en 7 ríos tributarios del PNSN, Mérida, Venezuela

TABLE 1

Environmental measures in 7 tributaries rivers of the PNSN, Merida, Venezuela

\begin{tabular}{clccccccc}
$\begin{array}{c}\text { Número } \\
\text { del río }\end{array}$ & \multicolumn{1}{c}{ Río } & $\begin{array}{c}\text { Uso de la } \\
\text { tierra }\end{array}$ & $\begin{array}{c}\text { Altitud } \\
(\mathrm{msnm})\end{array}$ & $\begin{array}{c}\text { Temp. Amb. } \\
\left({ }^{\circ} \mathrm{C}\right)\end{array}$ & $\begin{array}{c}\text { Profundidad } \\
(\mathrm{cm})\end{array}$ & $\begin{array}{c}\text { Velocidad } \\
(\mathrm{m} / \mathrm{s})\end{array}$ & $\begin{array}{c}\text { Temp } \\
\text { Agua } \\
\left({ }^{\circ} \mathrm{C}\right)\end{array}$ & $\begin{array}{c}\text { Tipo de } \\
\text { sustrato }\end{array}$ \\
1 & Aracay & $\mathrm{R}$ & $1815 \pm 16$ & 24 & 30 & 0.2 & 17.6 & $\mathrm{P} / \mathrm{Ro} / \mathrm{G}$ \\
2 & Santo Domingo & $\mathrm{B}$ & $3470 \pm 15$ & 26 & 15 & 0.43 & 9.1 & $\mathrm{P} / \mathrm{Ro}$ \\
3 & Corcovada & $\mathrm{U}$ & $3509 \pm 6$ & 18 & 10 & 0.25 & 9.5 & $\mathrm{P} / \mathrm{Ro}$ \\
4 & Las Piñuelas & $\mathrm{C}$ & $3240 \pm 12$ & 17 & 30 & 0.21 & 9.1 & $\mathrm{P} / \mathrm{A} / \mathrm{G}$ \\
5 & La Mucuy & $\mathrm{Rp}$ & $1808 \pm 12$ & 15 & 60 & 0.5 & 12 & $\mathrm{P} / \mathrm{G}$ \\
6 & La Picón & $\mathrm{B}$ & $2266 \pm 16$ & 20 & 33.3 & 0.2 & 12.3 & $\mathrm{P} / \mathrm{Ro}$ \\
7 & Nuestra Señora & $\mathrm{B}$ & $2348 \pm 12$ & 30 & 57 & 0.6 & 13.2 & $\mathrm{P} / \mathrm{Ro}$
\end{tabular}

Donde $\mathrm{Rp}=$ represa; $\mathrm{B}=$ bosque: $\mathrm{U}=$ urbano; $\mathrm{C}=$ comercial (ganadería, agricultura); $\mathrm{R}=$ recreacional; $\mathrm{P}=$ piedra; $\mathrm{G}=\mathrm{grava}$; $\mathrm{Ro}=$ roca; $\mathrm{A}=$ arena. 
CUADRO 2

Variables químicas de los 4 meses de muestreo (Febrero, Marzo, Abril y Mayo de 2009)

TABLE 2

Chemical variables for 4 months of sampling (February, March, April and May 2009)

\begin{tabular}{|c|c|c|c|c|c|c|c|}
\hline & 1 & 2 & 3 & 4 & 5 & 6 & 7 \\
\hline $\mathrm{pH}$ & $8.6 \pm 0.1$ & $8.3 \pm 0.1$ & $8.9 \pm 0.1$ & $8.6 \pm 0.1$ & $8.2 \pm 0.1$ & $8.9 \pm 0.1$ & $8.7 \pm 0.1$ \\
\hline Salinidad (ppm) & $40 \pm 0.1$ & 0 & $10 \pm 0.1$ & $10 \pm 0.1$ & $120 \pm 1.1$ & $30 \pm 0.9$ & $20 \pm 0.5$ \\
\hline Conductividad (uS/cm) & $79.4 \pm 0.1$ & $16.4 \pm 0.1$ & $31.6 \pm 1.4$ & $35.4 \pm 1.1$ & $23.8 \pm 1.0$ & $63.1 \pm 0.1$ & $59.7 \pm 1.1$ \\
\hline TDS (ppm) & $50 \pm 1.1$ & $10 \pm 0.1$ & $20 \pm 0.4$ & $20 \pm 0.4$ & $70 \pm 1.1$ & $40 \pm 1.0$ & $40 \pm 1.0$ \\
\hline Dureza (gpg $\mathrm{CaCO}_{3}$ ) & $3.48 \pm 0.4$ & $1.16 \pm 0.1$ & $1.16 \pm 0.1$ & $2.61 \pm 0.2$ & $6.96 \pm 0.6$ & $3.48 \pm 0.1$ & $4.2 \pm 0.5$ \\
\hline Oxígeno disuelto (ppm) & $14 \pm 0.2$ & $14 \pm 0.2$ & $6 \pm 0.1$ & $6 \pm 0.1$ & $2 \pm 0.1$ & $5 \pm 0.1$ & $5 \pm 0.1$ \\
\hline Nitrito (ppm) & $0.5 \pm 0.1$ & $1 \pm 0.1$ & 0 & 0 & 0 & 0 & 0 \\
\hline Nitrato (ppm) & $5 \pm 0.1$ & 0 & 0 & 0 & 0 & 0 & 0 \\
\hline
\end{tabular}

Los valores señalan la media aritmética. Donde: 1. Río Aracay, 2. Río Santo Domingo, 3. Quebrada Corcovada, 4. Quebrada Las Piñuelas, 5. Río La Mucuy, 6. Río La Picón, 7. Río Nuestra Señora. Se reportan medidas y desviación estándar.

The values indicate the arithmetic mean. Where: 1 . Aracay river, 2. Santo Domingo river, 3. Corcovada river, 4. Las Piñuelas river, 5. La Mucuy river, 6. La Picon river, 7. Nuestra Señora river. Report measurements and standard deviation are show.

presentaron características químicas similares y con la ausencia de una fuente de perturbación humana o natural permanente.

Distribución de los plecópteros: En sólo tres ríos (Nuestra Señora, La Picón y Corcovada) se encontró la presencia de plecópteros, con un total de 135 individuos de cuatro especies del género Anacroneuria. La especie con mayor número de individuos fue Anacroneuria chorrera (55), seguida de A. cacute (37), A. tachira (23) y A. paleta (20). La distribución de las poblaciones de plecópteros en los 3 ríos se muestra a continuación:

1. Río Nuestra Señora: individuos de la especie A.paleta Stark, con un total del $11,11 \%$ del total de plecópteros recolectados para todas las localidades. Es el primer registro de la especie en este río.

2. Río La Picón: Se recolectaron cuatro especies diferentes: A. paleta Stark, A. cacute Stark y Maldonado, A. chorrera Stark y $A$. tachira Stark, con un total de $69.44 \%$ del total de plecópteros recolectados para todas las localidades. Esta localidad resultó con la mayor diversidad y cantidad de especies de plecópteros recolectados.

3. Quebrada Corcovada: individuos de la especie A. chorrera Stark, con un total del 19,44\% del total de plecópteros recolectados para todas las localidades. Este es el primer reporte de esta especie para este río.

\section{Caracterización de las ninfas:}

A. paleta Stark. Stark 1995:236. Holotipo $\sigma^{7}$, para el río Santo Domingo y Paratipos para Barinas, Barinitas y Mérida. Maldonado et al. 2002: 227 (ninfa), Gamboa et al. 2009a: 232 (relaciones tróficas), Gamboa et al. 2009b: 232 (composición de la dieta), Gamboa et al. (en prep.) y Gamboa \& Arrivillaga (en prensa).

A. cacute Stark y Maldonado. Maldonado et al. 2002:223. Holotipo ơ', Río La Picón y Paratipos para Mérida. Maldonado 2002 (ninfas). Gamboa et al. 2009a: 232 (relaciones tróficas), Gamboa et al. 2009b: 232 (composición de la dieta), Gamboa et al. (en prep.) y Gamboa $\&$ Arrivillaga (en prensa).

A. tachira Stark. Maldonado 2002 ( $q \bigcirc^{7}$ figs., immaduros). Stark y Zuñiga 2002: 6 (q figs.). 
Holotipo y Paratipo, Río El Valle para Táchira. Gamboa et al. 2009a: 232 (relaciones tróficas), Gamboa et al. 2009b: 232 (composición de la dieta), Gamboa et al. (en prep.) y Gamboa \& Arrivillaga (en prensa).

A. chorrera Stark. Stark 1995: 17 ( figs.). Holotipo y Paratipos, Río La Chorrera para Mérida. Maldonado 2002 (Q ○७ figs., inmaduros). Gamboa et al. 2007: 30 (morfometría geométrica de la pteroteca). Gamboa et al. 2009a: 232 (relaciones tróficas), Gamboa et al. 2009b: 232 (composición de la dieta), Gamboa et al. (en prep.) y Gamboa \& Arrivillaga (en prensa).

Las ninfas recolectadas en el río La Picón presentaron tamaño corporal y peso seco similar (Cuadro 3), excepto los individuos de la especie A. paleta, la cual es de menor tamaño corporal comparada con el resto de especies. No se encontraron diferencias significativas entre las medidas (Prueba T-student, $\mathrm{p}>0,150$, $\mathrm{n}=135$ ).

Relaciones ambientales: Los análisis de componentes principales (Fig. 2), muestran que los ríos Nuestra Señora y Corcovada presentan caracteres ambientales similares, donde la caracterización del hábitat, la velocidad de la corriente, el oxígeno disuelto y la ausencia de fuentes de perturbación antrópica, los caracteriza como un sitio idóneo para el establecimiento de poblaciones de plecópteros.

\section{DISCUSIÓN}

La distribución de los plecópteros en zonas templadas ha demostrado que está influenciada por el régimen termal, la composición del sedimento y la disponibilidad del alimento (Mavri et al. 2000). Y en zonas tropicales: por la altitud, el sustrato, la magnitud del curso de agua (Vidal \& Membiela 1993, Diniz-Filho et al. 1998) y el efecto antrópico (Bispo \& Oliveira 1998, Bispo et al. 2002, Froehlich \& Oliveira 2002).

Los resultados sugieren que las poblaciones de plecópteros están influenciadas directamente por las actividades antrópicas, más que por algún otro cambio ecológico, tal como sugiere Froehlich y Oliveira (2002) para plecópteros en Brasil. El establecimiento de parques de recreación y zonas urbanas, altera las condiciones del cauce que ocasiona la extinción o reducción de la distribución de estos insectos, como consecuencia de su sensibilidad a los cambios del cauce.

Una fuerte reducción o desaparición de las poblaciones de plecópteros, fue observada para los ríos Santo Domingo y Aracay. En este estudio, ningún individuo fue encontrado en el cauce de estos ríos, probablemente como consecuencia de la presencia de nitrito y nitrato producto de la ganadería y agricultura, lo que ocasiona una alta concentración de oxígeno disuelto en el agua de estos ríos andinos debido

CUADRO 3

Medidas corporales, peso seco y desviación estándar de individuos de Anacroneuria

TABLE 3

Body measurements, dry weight and standard deviation of individuals of Anacroneuria

\begin{tabular}{llccc}
\multicolumn{1}{c}{ Ríos } & \multicolumn{1}{c}{ Especie $(\mathrm{N})$} & Ancho cápsula cefálica $(\mathrm{mm})$ & Largo total $(\mathrm{mm})$ & Peso seco $(\mathrm{mg})$ \\
Nuestra Señora & A. paleta $(7)$ & $2.3 \pm 0.5$ & $11.6 \pm 1.4$ & $3.9 \pm 0.59$ \\
La Picón & A. chorrera $(39)$ & $3.5 \pm 0.4$ & $12.1 \pm 1.4$ & $4.1 \pm 0.59$ \\
& A. paleta $(16)$ & $1.1 \pm 0.5$ & $5.0 \pm 0.5$ & $0.5 \pm 0.6$ \\
& A. cacute $(37)$ & $2.5 \pm 0.5$ & $11.6 \pm 1.4$ & $3.9 \pm 0.5$ \\
Corcovada & A. tachira $(23)$ & $2.7 \pm 0.7$ & $12.2 \pm 1.0$ & $4.0 \pm 0.5$ \\
& A. chorrera $(13)$ & $2.7 \pm 0.7$ & $10.7 \pm 0.3$ & $3.9 \pm 0.46$
\end{tabular}




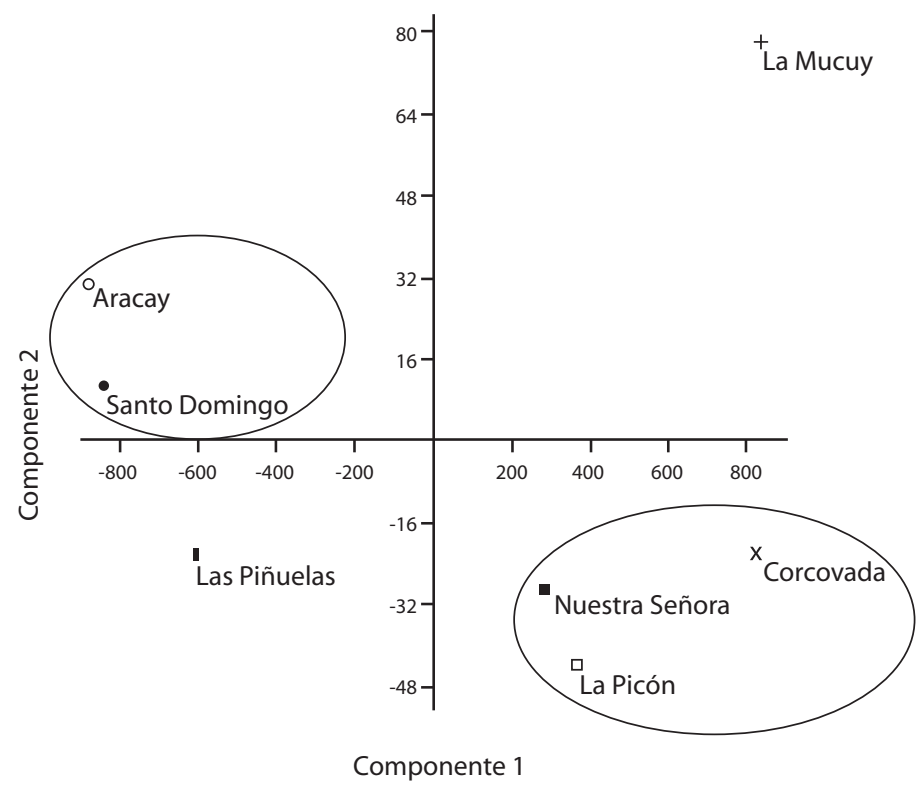

Fig. 2. Análisis de componentes principales de siete ríos en el Parque Nacional Sierra Nevada, Mérida, Venezuela, basado en las medidas físicas y las variables químicas. El componente 1 y 2 representan el $99.38 \%$ y $0.318 \%$ de la variabilidad, respectivamente.

Fig. 2. Principal component analysis of seven rivers in the Sierra Nevada National Park in the state of Merida, Venezuela, based on physical measurements and chemical variables. Component 1 and 2 represent $99.38 \%$ and $0.318 \%$ of the variability, respectively.

a la frecuente ruptura de puentes de hidrógeno (Miserendino 2006) y por ende al cambio de condiciones favorables para la vida de estos insectos, ya que altas concentraciones de oxígeno disuelto son letales para los plecópteros (Benedetto 1970).

Igualmente, el cambio ecológico del río Santo Domingo es consecuencia de la construcción de la represa: "José A. Páez", que conllevó a la canalización del río. Cressa y Stark (2003), señalan que en este río se encontraron 6 especies de plecópteros del género Anacroneuria (A. chorrera, A. cuadrada, A. paleta, A. digitata, A. bari, A. caraca). La reducción y cambio del hábitat ribereño producto de la canalización del cauce, al igual que la ganadería (observación personal) en sus alrededores, ocasiona que las poblaciones de plecópteros se encuentren en extinción o en peligro de supervivencia. En algunos sectores de Europa, los plecópteros han sido completamente exterminados debido a la gradual fragmentación del hábitat, contaminación y pérdida de biotipos consecuentes de la construcción de represas (Küry 1997, Landa et al. 1997).

La alta influencia de agricultura y ganadería de montaña ocasiona la interrupción del cauce debido al riego o posible fuente de contaminación por desagüe de aguas servidas a ríos cercanos (Mavri et al. 2000), el río Aracay, cercano a la población "Las Piedras", es un ejemplo claro de este tipo de influencia antrópica.

La intervención humana también influyó en la desaparición de poblaciones de plecópteros en los ríos La Mucuy y Las Piñuelas, como consecuencia de actividades recreacionales (balnearios, montañismo, hoteles, etc.). 
Anteriormente en el estudio de Maldonado (2002) y Cressa \& Stark (2003), se reportaron 2 especies de plecópteros para La Mucuy ( $A$. chorrera, A. fenestrata) y 1 especie para Las Piñuelas (A. chorrera).

Bajo el mismo estudio realizado por Maldonado (2002), se reporta para el río La Picón, 5 especies del género Anacroneuria (A. chorrera, A. cacute, A. tachira, A. paleta, A. muesca). Estudios anteriores de Gamboa et al. (2009a, b) señalan la presencia de sólo 4 de ellas $(A$. chorrera, A. cacute, A. tachira, A. paleta) para el mismo río, argumentando poca densidad de la especie A. muesca, como característica intrínseca de la misma, lo que explicaría porque no es encontró en el cauce. Tal como muestra este estudio, sólo 4 especies fueron encontradas, implicando una posible desaparición de la especie A. muesca.

Las especies de plecópteros se encuentran a veces sólo en un sistema de corriente en particular o se consideran raras y restringidas a una región geográfica muy pequeña, lo que resulta la necesidad de una especial consideración y estudio (Stewart \& Stark 2002). No se conoce hasta ahora posibles causas de la no aparición de A. muesca, pero es viable que cambios ambientales influyeran en la densidad de plecópteros. Maldonado (2002) señala que la especie presenta una escala de temperatura del agua entre 9.5 a $22^{\circ} \mathrm{C}$, y reporta que para el 2000 la temperatura del agua máxima alcanzada para el río La Picón fue $28^{\circ} \mathrm{C}$ y para el 2001 fue de $30^{\circ} \mathrm{C}$, lo que implicó el aumento de $2^{\circ} \mathrm{C}$ en sólo un año.

Kanzaci y Dügel (2008) establecen modelos climáticos para predecir como el aumento de $2^{\circ} \mathrm{C}$ de temperatura afecta a los insectos acuáticos, por lo que se concluye que el aumento de sólo un grado de temperatura eliminaría a las especies de insectos acuáticos más sensibles. En este estudio no se presenta la temperatura máxima alcanzada, sólo la temperatura media de $12.3^{\circ} \mathrm{C}$ (Cuadro 1), por lo que no puede obtener conclusiones concretas, pero deja una interrogante para futuros trabajos.

Para los restantes ríos muestreados, Nuestra Señora y la quebrada Corcovada, se establece el primer registro de presencia de plecópteros en el cauce (A. paleta y A. chorrera, respectivamente), donde los holotipos son del río $\mathrm{La}$ Chorrera y El Valle, para el estado Mérida y Táchira, respectivamente.

La presencia de plecópteros para tres ríos muestreados (Nuestra Señora, La Picón y La Corcovada), permite observar que características ambientales similares, como el tipo de sustrato, velocidad de la corriente, el $\mathrm{pH}$, oxígeno disuelto, la disponibilidad del alimento y la conservación de bosques ribereños (observación personal), permiten: el suministro de materia orgánica y el aporte de nutrientes para el hábitat de los insectos (Wiberg-Larsen et al. 2000), la colonización y establecimiento de poblaciones de plecópteros y de insectos acuáticos en general (Vidal \& Membiela 1993, Mavri et al. 2000, Froehlich \& Oliveira 2002) y el estudio de la buena calidad ecológica (Miserendino 2000) del cauce.

A pesar de ser un hábitat idóneo para estos insectos, la biomasa de las poblaciones de plecópteros, especialmente la especie A. paleta, son bajas, comparados con los valores reportados por Cressa (1999) para el género Anacroneuria en el río Orituco, Gamboa y Castillo (2009) para el mismo género en el río Guare y los promedios de Gamboa y Arrivillaga (en prensa) para plecópteros del estado Mérida en Venezuela. Lo que puede indicar una alarma de posible peligro para la supervivencia de poblaciones de plecópteros que habitan el PNSN.

Los planes de conservación de estos ríos son necesarios para beneficiar tanto a las poblaciones humanas como animales y vegetales, por lo tanto, es necesario evitar establecer zonas recreacionales o económicas cercas de los ríos donde fue establecida la presencia de plecópteros, así mismo, evitar el desagüe de contaminación orgánica del agua por parte de sectores poblados cercanos a los ríos. Es necesario mantener de manera prístina el acceso al río así como sus alrededores, para que las poblaciones de plecópteros permanezcan y re-colonicen de manera natural afluentes cercanos.

Las larvas de plecóptera tienen un hábitat y necesidades de agua potable similares a 
las pequeñas truchas y otros peces de tamaño parecido (Stewart \& Stark 2002), por lo que las técnicas utilizadas para mantener saludables las poblaciones de peces nativos podrían funcionar adecuadamente para promover las poblaciones de larvas.

Las poblaciones de plecópteros se hallan en peligro de desaparición e inclusive se han llegado a extinguir en gran parte de su área de distribución como consecuencia de actividades humanas (ganadería, agricultura, represas) (Stewart \& Stark 2002). La destrucción del hábitat afecta a la calidad del agua y representan la mayor amenaza para los plecópteros. Por lo que, el aumento de estudios de este grupo de insectos y su hábitat conllevará a entender la dinámica del río así como el establecimiento de poblaciones de plecópteros endémicos para desarrollar planes de conservación.

\section{AGRADECIMIENTOS}

Este estudio ha sido financiado por Provita bajo el programa de Iniciativa de Especies Amenazadas con el número 2009-33. Extiendo mi agradecimiento a Raúl Vegas y Nicolás Hatcher por su asistencia en el campo y al Laboratorio de Limnología y a María Mercedes Castillo de la Universidad Simón Bolívar por el préstamo de equipos.

\section{RESUMEN}

La distribución longitudinal de las especies de plecópteros fue examinada a lo largo del Parque Nacional Sierra Nevada en la región Andina del estado Mérida, Venezuela. El PNSN es uno de las más grandes regiones protegidas y abarca dos subcuencas hidrográficas importantes. Muestras cuantitativas fueron recolectadas en 7 ríos tributarios de estas cuencas a lo largo del PNSN desde febrero a mayo del 2009, con un total de 135 individuos de 4 especies del género Anacroneuria. Sólo en tres ríos (Nuestra Señora, La Picón y Corcovada) se encontró la presencia de plecópteros, los análisis de componentes principales muestran que la caracterización del hábitat, la velocidad de la corriente, el oxígeno disuelto y la ausencia de fuentes de perturbación antrópica influyen en un hábitat idóneo para las poblaciones. Los plecópteros se hallan en peligro de desaparición o incluso se han llegado a extinguir en gran parte de su área de distribución como consecuencia de actividades huma- nas. Planes de conservación deben ser implementados con urgencia, como evitar zonas recreacionales y/o económicas en las cercanías de los mismos.

Palabras clave: ríos Andinos, Anacroneuria, gradiente longitudinal, relaciones ambientales, actividades humanas.

\section{REFERENCIAS}

Albariño, R.J. 1997. Spatial distribution of Plecoptera from an Andean-Patagonic lotic environment in Argentina. Rev. Bras. Biol. 57: 629-636.

Allan, D.J. 1995. Stream Ecology. Structure and Function of running water. Chapman \& Hall, Londres, Inglaterra.

Benedetto, L. 1970. Observations on the oxygen needs of some species of european Plecoptera. Int. Revue ges. Hydrobiol. 55: 505-510.

Bispo, P.C. \& L.G. Oliveira. 1998. Distribuição espacial de insetos aquáticos (Ephemeroptera, Plecoptera e Trichoptera), em córregos de cerrado do Parque Ecológico de Goiânia, Estado de Goiás, p. 175-189 In J.L. Nessimian \& A.C. Carvalho (eds.). Ecologia de insetos aquáticos. Oecol. Bras., UFRJ, Rio de Janeiro.

Bispo, P.C., C.G. Froehlich \& L.G. Oliveira. 2002. Spatial distribution of Plecoptera nymphs in streams of a Mountainous area of central Brazil. Braz. J. Biol. 62: 409-417.

Brittain, J. 1991. Life history characteristics as a determinant of the response of mayflies and stoneflies to man-made environmental disturbance (Ephemeroptera and Plecoptera), p. 539-545. In J. Alba-Tercedor \& A. Sanchez Ortega (eds.). Overview and Strategies of Ephemeroptera and Plecoptera. Sandhill Crane. Gainesville, Florida, EEUU.

Cressa, C. 1999. Dry mass estimates of some tropical aquatic insects. Rev. Biol. Trop. 47: 133-141.

Cressa, C. \& B. Stark. 2003. Plecópteros, p. 478-487. In M. Aguilera, A. Azocar \& E. González (eds.). Biodiversidad de Venezuela. Fundación Polar y Ministerio de Ciencia y Tecnología. Exlibris, Caracas, Venezuela.

Cummins, K.W. \& M.J. Klug. 1979. Feeding ecology of stream invertebrates. Ann. Rev. Ecol. Syst. 10: 147-172.

Death, R.G. \& M.J. Winterbourn. 1995. Diversity patterns in stream benthic invertebrate communities: the influence of habitat stability. Ecology 76: 14461460 . 
Diniz-Filho, J.A.F., L.G. Oliveira \& M.M. Silva. 1998. Explaining the beta diversity of aquatic insects in "cerrado" streams from Central Brazil using multiple Mantel Test. Rev. Bras. Biol. 58: 223-231.

Domínguez, E. \& J.M.B. Valdez. 1992. Altitudinal replacement of Ephemeroptera in a subtropical river. Hydrobiologia 246: 83-88.

Dudgeon, D. 1993. The effects of spate-induced disturbance, predation and environmental complexity on macroinvertebrates in a tropical stream. Freshwater Biol. 30: 189-197.

Froehlich B.P. \& L.G. Oliveira. 2002. Spatial distribution of Plecoptera nymphs in streams of a mountainous area f Central Brazil. Braz. J. Biol. 62: 409.417

Fundación Andígena. 2009. Parque Nacional Sierra Nevada. Mérida, Venezuela. (Consultado: octubre 2009, http://sierranevada.andigena.org/)

Gamboa, M., R. Cipriani \& J. Arrivillaga. 2007. Ontogenia de Anacroneuria chorrera (Plcoptera:Perlidae) usando morfometría geométrica de la pteroteca. Entomol Mex. 6: 183-188.

Gamboa, M. \& M.M. Castillo. 2009. Primer registro de ninfas de Anacroneuria (Plecoptera:Perlidae) para el río Guare en Venezuela. Bol. Cent. Invest. Biol. 43: 405-410.

Gamboa, M., M.M. Chacon \& S. Segnini. 2009a. Ritmo diario de alimentación y tamaño de presa en cuatro especies simpátridas de Plecoptera (Insecta) en un río tropical Andino. Ecotropicos 22: 37-43.

Gamboa, M., M.M. Chacón \& S. Segnini. 2009b. Diet composition of the mature larvae of four Anacroneuria species (Plecoptera: Perlidae) from the Venezuelan Andes. Aquat. Insects 31: 409-417.

Giller, P.S. \& B. Malmqvist. 1998. The Biology of Streams and Rivers. Biology of Habitats. Oxford, Oxford, Inglaterra.

Hammer, O., D.A.T. Harper \& P.D. Ryan. 2001. PAST: Paleontological Statistics Software Package for Education and Data Analysis. Version 1.42. Palaeontologia Electronica 4: 1-9 (también disponible en línea: http://palaeo-electronica.org/2001_1/past/issue1_01. htm).

Harding, J.S., J.M. Quinn \& C.W. Hickey. 2000. Effects of mining and production forestry, p. 230-259. In K.J. Collier \& M.J. Winterbourn (eds.). New Zealand Stream Invertebrates: Ecology and Implications for Management. New Zealand Limnological Society. Christchurch.
Hurtado, S., F. García-Trejo \& P.J. Gutiérrez. 2005. Importancia ecológica de los macroinvertebrados bentónicos de la subcuenca del río San Juan, Querétaro, México. Folia Entomol. Mex. 44: 271-286.

Hynes, H.B.N. 1970. The ecology of stream insects. Ann. Rev. Entomol. 15: 25-42.

IUCN. 2009. IUCN Red List of Threatened Species. Cambridge CB3 0DL, Inglaterra. (Consultado: octubre 2009, www.iucnredlist.org).

Kazanci, N. \& M. Dügel. 2008. Prediction of global climate change impact on structure of aquatic insect assemblages by using species optimum and tolerance values of temperature. Rev. Hidrol. 2: 73-80.

Küry, D. 1997. Changes in the Ephemeroptera and Plecoptera population of a Swiss Jura stream (Roserenbach) between 1935 and 1990, p. 296-301. In P. Landolt \& M. Sartori (eds.). Ephemeroptera and Plecoptera: Biology, Ecology and Systematic. Fribourg, Suiza.

Landa, V.J., J. Helesic, T. Soldán \& S. Zahradkova. 1997. Stoneflies (Plecoptera) of the river Vltava, Czech Republic: A century of Extincion, p. 288-295. In P. Landolt \& M. Sartori (eds.). Ephemeroptera \& Plecoptera. Biology-Ecology-Systematics. MTL, Fribourg, Suiza.

Lillehammer, A. \& J.E. Brittain. 1987. Longitudinal zonation of the benthic invertebrate fauna in the River Glomma, Eastern Norway. Fauna Norv. Ser. A 8: $1-10$.

López, M.V. \& P. Membiela. 1993. Algunos factores abióticos de distribución de los Efemerópteros y Plecópteros (Insecta) en las Sierras Segundera, Cabrera y Teleno (NW Península Ibérica). Limnética 9: 99-106.

Maldonado, V. 2002. Biodiversidad de plecópteros (Insecta: Plecoptera: Perlidae) en Venezuela. Tesis doctoral, Universidad Central de Venezuela, Caracas, Venezuela.

Maldonado, V., B.P. Stark \& C. Cressa. 2002. Descriptions and records of Anacroneuria from Venezuela (Plecoptera:Perlidae). Aquat. Insects 24: 219-236.

Margalef, R. 1983. Limnología. Omega, Barcelona, España.

Mavri, U., S. Podgornik \& I. Sivec. 2000. Influence of some ecological factors on the distribution of Plecoptera in a small subalpine stream. Verh. Internat. Verein. Limnol. 27: 981-984. 
Miserendino, M.L. 2000. Distribución y variación estacional de Plecoptera en un sistema fluvial de la cordillera patagónica. Rev. S. Entomol. Argent. 59: 149-160.

Miserendino, M.L. 2006. Seasonal and spatial distribution of stoneflies in the Chubut River (Patagonia, Argentina). Hidrobiologia 568: 263-274.

Merritt, R.W. \& K.W. Cummins. 1996. An introduction to the aquatic insects of North America. Kendall-Hunt, Dubuque, Iowa, EEUU.

Peckarsky, B.L. 1993. Sublethal consequences of streamdwelling predatory stoneflies on mayfly growth and fecundity. Ecology 74: 1836-1846.

Pérez, R. 2000. Interpretación ecológica de la ganadería extensiva y sus interrelaciones con la agricultura en el Piso Agrícola del Páramo de Gaviria. Andes Venezolanos. Tesis Maestría, Universidad de Los Andes, Mérida, Venezuela.

Rabeni, C.F. \& G.W. Minshall. 1977. Factors affecting micro-distribution of stream benthic insects. Oikos 29: 33-43.

Reice, S.R. 1980. The role of substratum in benthic macroinvertebrate micro-distribution and litter decomposition in a woodland stream. Ecology 6: 580-590.

Resh, V.H., A.V. Brown, A.P. Covich, M.E. Gurtz, H.G. Li, G.W. Minshall, S.R. Reice, A.L. Sheldon, J.B. Wallace \& R.C. Wissmar. 1988. The role of disturbance in stream ecology. J. N. Am. Benthol. Soc. 7: 433-455.

Segnini, S. 2003. El uso de los macroinvertebrados bentónicos como indicadores de la condición ecológica de los cuerpos de agua corriente. Ecotropicos 16: 45-63.

Scarsbrook, M.R. \& J. Halliday. 1999. Transition from pasture to native forest land-use along stream continua: effects on stream ecosystems and implications for restoration. New Zeal. J. Mar. Fresh. 33: 293-310.
Stark, B.P. 1995. New species and records of Anacroneuria (Klapálek) from Venezuela. Spixiana 18: 211-249.

Stark, BP. 1999. Anacroneuria from northeastern South America (Insecta:Plecoptera:Perlidae). P. Biol. S. Wash. 112: 70-93.

Stark, B.P. 2000. Notes on the Anacroneuria (Plecoptera:Perlidae) of Guayana with the description of a new species. Aquat. Insects 22: 305-310.

Stark, B.P. \& M. Zúñiga. 2002. The Anacroneuria guambiana complex of South America (Plecoptera: Perlidae), p. 229-237 In E. Gaino (ed.). Proceedings of the International Symposium of Plecoptera. Italia.

Statzner, R.B., J.A. Gore \& V.H. Resh. 1988. Hydraulic stream ecology: observed patterns and potential applications. J. N. Amer. Benthol. Soc. 7: 307-360.

Stewart, K.W. \& B.P. Stark. 2002. Nymphs of North American stonefly genera (Plecoptera). The Caddis, Columbus, Ohio, EEUU.

Townsend, C.R., C.J. Abruckle, T.A. Crowl \& M.R. Scarsbrook. 1997. The relationship between land use and physicochemistry, food resources and macroinvertebrate communities in tributaries of the Taieri River, New Zealand: a hierarchically scaled approach. Freshwater Biol. 37: 177-191.

Vidal, M. \& P. Membiela. 1993. Algunos factores abióticos de distribución de los efemerópteros y plecópteros (Insecta) en las Sierras Segundera, Cabrera y Teleno (NW Península Ibérica). Limnética 9: 99-106.

Ward, J.V. \& J.A. Stanford. 1982, Thermal responses in the evolutionary ecology of aquatic insects. Ann. Rev. Entomol. 27: 97-117.

Wiberg-Larsen, P., K.P. Brodersen, S. Birkholm, P.N. Grons \& J. Skriver. 2000. Species richness and assemblage structure of Trichoptera in Danish streams. Freshwater Biol. 43: 633-647. 